\title{
A spring-dashpot system for modelling lung tumour motion in radiotherapy
}

\author{
P.L. Wilson ${ }^{\mathrm{a} *}$ and J. Meyer ${ }^{\mathrm{b}}$ \\ ${ }^{a}$ Department of Mathematics and Statistics, University of Canterbury, Christchurch, New Zealand; \\ ${ }^{b}$ Department of Physics and Astronomy, University of Canterbury, Christchurch, New Zealand
}

(Received 6 August 2008; final version received 20 October 2008)

\begin{abstract}
A 3D system of springs and dashpots is presented to model the motion of a lung tumour during respiration. The main guiding factor in configuring the system is the spatial relationship between abdominal and lung tumour motion. A coupled, non-dimensional triple of ordinary differential equations models the tumour motion when driven by a 3D breathing signal. Asymptotic analysis is used to reduce the system to a single equation driven by a 3D signal, in the limit of small lateral and transverse tumour motions. A numerical scheme is introduced to solve this equation, and tested over wide parameter ranges. Real clinical data is used as input to the model, and the tumour motion output is in excellent agreement with that obtained by a prototype tumour tracking system, with model parameters obtained by optimization. The fully 3D model has the potential to accurately model the motion of any lung tumour given an abdominal signal as input, with model parameters obtained from an internal optimization routine.
\end{abstract}

Keywords: modelling; asymptotic analysis; numerical analysis; optimization; lung; tumour; respiration; radiotherapy

AMS Subject Classification: 92C05; 92C10; 92C50

\section{Introduction}

Cancer of the respiratory tract is a common disease with relatively poor prognosis. The leading cause of death from cancer in 2004 in New Zealand was cancer of the trachea, bronchus and lung. It ranked 3rd and 4th in terms of incidence for males and females, respectively, and the 1- and 5-year survival is approximately 30 and 15\% [9]. Radiation therapy alone or in combination with chemotherapy and/or surgery are the main modalities to treat cancer. One of the reasons for the unfavourable treatment response to radiation therapy is the fact that for lesions in the lung comparatively large treatment margins are required to account for respiratory-induced tumour motion [6]. Inevitably, this results in a larger volume of normal tissue being irradiated, thereby considerably increasing the side effects of the treatment. Consequently, the total tolerable treatment dose that can be given to the tumour is lower than what is necessary for adequate tumour control.

If the exact tumour position were known at all times during irradiation, the treatment parameters could be adjusted and hence the dose delivered more conformally [4,7]. This, in turn, would make it possible for higher overall doses to be given to the tumour keeping the side effects at a comparable level. In practise, it is technically difficult to directly determine the

* Corresponding author. Email: p.wilson@math.canterbury.ac.nz 
actual tumour position non-invasively in real-time during treatment $[2,11,14,15,17,18,24]$. Instead, most non-invasive approaches have resorted to measuring the respiratory signal of the patient, which is ultimately related to lung tumour motion. Accurate and reliable models are required to correlate the relationship between respiratory and lung tumour motion to ensure that the position of the tumour is known at all times. Failure to determine irregular breathing or so-called base line drift can result in inaccurate dose delivery.

A thorough description of modelling lung tissue properties taking into account viscoelastic non-linear stress-strain behaviour can be found in Refs [19,20]. However, the focus of this work is the spatio-temporal relationship between abdominal motion and that of a tumour embedded in the lung tissue. Previous work investigated simple 'grey box' type models of this relationship [10]. While these models were successfully applied to clinical cases, intrinsically they do not have the ability to model certain behaviours of the tumourbreathing signal relationship, such as when the tumour trajectory exhibits a hysteresis [17] or when a spring-like elasticity can be observed. This is because they attempt to fit model parameters (in a least-squares sense) to a linear (or non-linear) model, which does not resemble the physical relationship of the data set. This is why they are referred to as 'black box' models, or, if some prior knowledge is incorporated, as 'grey box' models.

In this work, a novel 3D model is presented, which aims to physically model the spatial relationship between an external signal, representing abdominal breathing motion, and a lung tumour. The approach is based on an array of springs and dashpots arranged to model tumours at any location within the lung. While the mathematical model is general for any tumour in the lung, the parameters governing the springs and dashpots are patient- and tumour locationspecific. The characteristics of the model are analysed both mathematically and by means of computer simulations. Finally, the model is validated against real clinical tracking data.

\section{A full 3D model}

\subsection{Physical setup}

Figure 1 shows the 3D system of springs and dashpots at a general time $t^{*}$, where a superscript star indicates a dimensional quantity. The tumour lies at position $\boldsymbol{p}_{T}^{*}(t)=$ $\left(x_{T}^{*}\left(t^{*}\right), y_{T}^{*}\left(t^{*}\right), z_{T}^{*}\left(t^{*}\right)\right)$ where $\left(x^{*}, y^{*}, z^{*}\right)$ forms a right-handed triad as shown, with corresponding unit vectors $(\boldsymbol{i}, \boldsymbol{j}, \boldsymbol{k})$. Although we have drawn the tumour lying at negative $x^{*}$, $y^{*}$ and $z^{*}$, it will in general explore both positive and negative values of all directions. In so doing, each spring can if necessary move through its pivot point without penalty. The tumour is connected via spring $s_{X}$ of natural length $l_{X}^{*}$ to a pivot point free to move in the plane $x^{*}=l_{X}^{*}$. Dashpot $d_{X}$ provides damping of the $x^{*}$-motion, and is likewise free to move in a $\left(y^{*}, z^{*}\right)$-plane to track the $\left(y^{*}, z^{*}\right)$-components of the tumour position. This 'tracking' behaviour of the spring $s_{X}$ and dashpot $d_{X}$ is a phenomenological model of the multiple forces acting laterally on the tumour due to multiple attachment points and lung movement. The mechanical responses of the connective tissue are not directly modelled, but rather the response of the tumour to diaphragm motion, resulting in abdominal movement. In this initial approach, a serial spring-dashpot arrangement in each direction forms the basis of the model. The tumour is also connected via a spring $s_{Z}$ of natural length $l_{Z}^{*}$ to a pivot point free to move in the plane $z^{*}=l_{Z}^{*}$. Dashpot $d_{Z}$ provides damping of the $z^{*}$-motion, being free to move in an $\left(x^{*}, y^{*}\right)$-plane to track the $\left(x^{*}, y^{*}\right)$-components of the tumour position.

Spring $s_{Y}$ of natural length $l_{Y}^{*}$ connects the tumour to the point $p_{D}^{*}\left(t^{*}\right)$ which represents the diaphragm. The diaphragm point $\boldsymbol{p}_{D}^{*}\left(t^{*}\right)$ is assumed to move only along a superior-inferior, or sup-inf (head-to-feet), line through the origin; it is constrained to lie on the line $x^{*}=0$, $z^{*}=0$. It is the diaphragm point which drives the tumour motion by means of being itself 


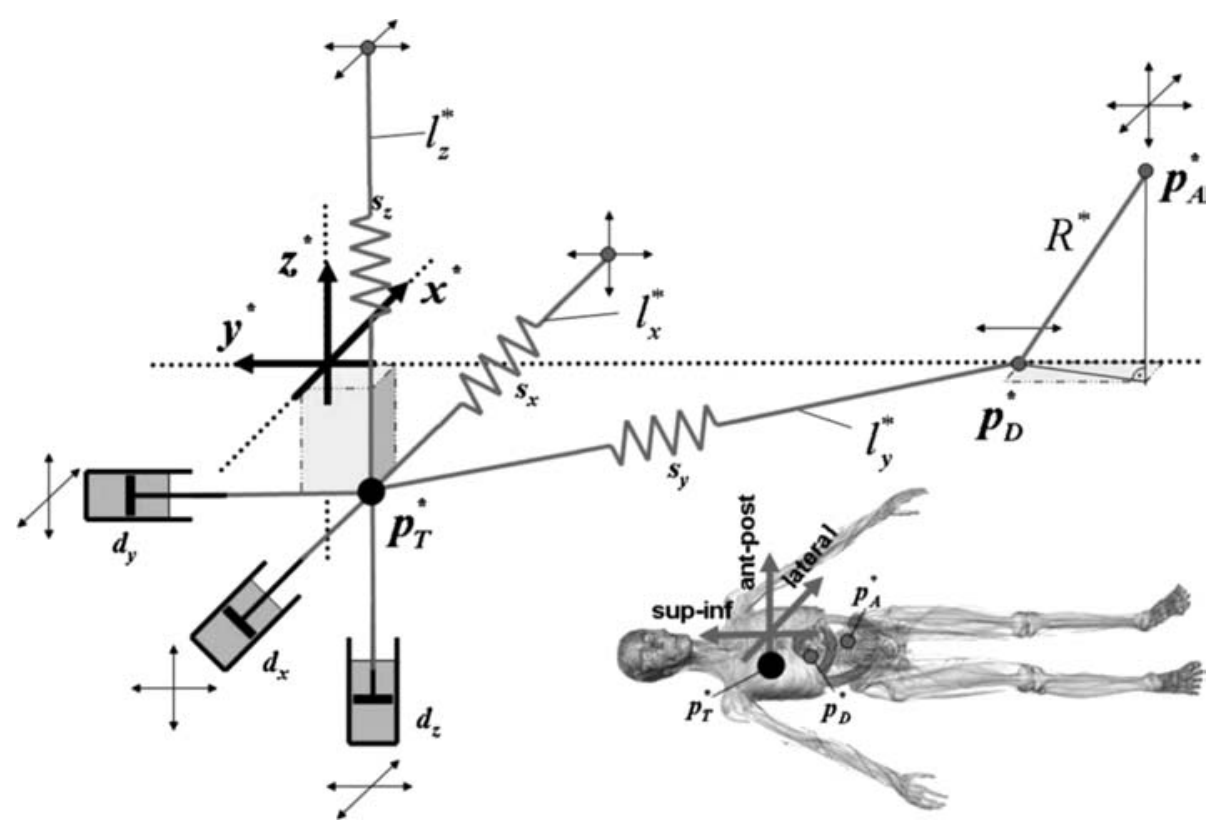

Figure 1. The spring-dashpot system. The coordinate axes, spring and dashpot labels, and the position of the tumour, diaphragm, and abdomen are shown. The degrees of freedom are indicated by arrows.

connected by a rigid rod of length $R^{*}$ to the point $\boldsymbol{p}_{A}^{*}\left(t^{*}\right)$, which models the time-varying abdominal position of the patient during breathing, and is in general 3D. Tension in the spring $s_{Y}$ acts only to change $\boldsymbol{p}_{T}^{*}$; the diaphragm position $\boldsymbol{p}_{D}^{*}$ varies only with changes in the abdomen position $\boldsymbol{p}_{A}^{*}$. Because both the tumour and abdomen each have three degrees of freedom we call this model the 3D-3D model referring to tumour and abdomen, respectively. (Experiments measure the abdominal motion, as described in Section 4, while diaphragm movement is not measured experimentally.) A dashpot $d_{Y}$ dampens the $y^{*}$-motion, and is free to move in an $\left(x^{*}, z^{*}\right)$-plane to track the $\left(x^{*}, z^{*}\right)$-components of the tumour motion.

We assume here that all springs follow Hooke's Law, with $s_{X}, s_{Y}, s_{Z}$ having stiffness $k_{X}^{*}, k_{Y}^{*}, k_{Z}^{*}$, respectively. Furthermore, we take all dashpots to provide friction proportional to the velocity component in the relevant direction. Again, other choices could be considered. Note that since the tumour motion is influenced by the mechanical properties of the lung tissue, future work will, if necessary, consider more complex spring-dashpot arrangements, non-Hookean spring behaviour, or alternative dampers.

In the next section we derive the coupled equations of motion which govern the 3D3D system.

\subsection{Governing equations}

Referring again to Figure 1 , we denote by $\theta_{X}$ the angle which $s_{Y}$ makes with the plane $x=0$, and the angle which $s_{Y}$ makes with the plane $z=0$ is denoted $\theta_{Z}$. These angles can be written in terms of the other variables. Then if the constant mass of the tumour is $m^{*}$, Newton's second law with the assumptions of Hookean springs and the form of the dashpot resistance gives the governing equations.

$$
m^{*} \ddot{x}_{T}^{*}=k_{X}^{*} x_{T}^{*}+k_{Y}^{*} \xi_{Y}^{*} \sin \theta_{X} \cos \theta_{Z}-\beta_{X}^{*} \dot{x}_{T}^{*}
$$




$$
\begin{aligned}
& m^{*} \ddot{y}_{T}^{*}=-k_{Y}^{*} \xi_{Y}^{*} \cos \theta_{X} \cos \theta_{Z}-\beta_{Y}^{*} \dot{y}_{T}^{*}, \\
& m^{*} \ddot{z}_{T}^{*}=k_{Z}^{*} z_{T}^{*}+k_{Y}^{*} \xi_{Y}^{*} \sin \theta_{Z}-\beta_{Z}^{*} \dot{z}_{T}^{*},
\end{aligned}
$$

where

$$
\xi_{Y}^{*}=\left\{x_{T}^{* 2}+\left[y_{A}^{*}-y_{T}^{*}+\left(R^{* 2}-x_{A}^{* 2}-z_{A}^{* 2}\right)^{\frac{1}{2}}\right]^{2}+z_{T}^{* 2}\right\}^{\frac{1}{2}}-l_{Y}^{*},
$$

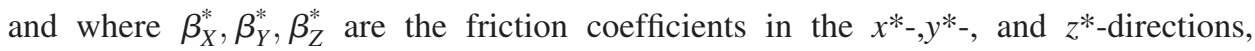
respectively, and a dot denotes differentiation with respect to time. These $3 \mathrm{D}$ equations are strongly coupled, and the forcing from the breathing appears explicitly in all three.

\subsubsection{Non-dimensional equations}

We proceed to non-dimensionalize the governing Equations (1-3); non-dimensional variables will be written without a superscript star. A representative length is taken to be $l_{Y}^{*}$, while $\tau^{*}$ denotes a general representative time scale. The non-dimensional equations are

$$
\begin{aligned}
& \ddot{x}_{T}=\omega_{X}^{2} x_{T}+\omega_{Y}^{2} \xi_{Y} \sin \theta_{X} \cos \theta_{Z}-2 \lambda_{X} \dot{x}_{T}, \\
& \ddot{y}_{T}=-\omega_{Y}^{2} \xi_{Y} \cos \theta_{X} \cos \theta_{Z}-2 \lambda_{Y} \dot{y}_{T}, \\
& \ddot{z}_{T}=\omega_{Z}^{2} z_{T}+\omega_{Y}^{2} \xi_{Y} \sin \theta_{Z}-2 \lambda_{Z} \dot{z}_{T},
\end{aligned}
$$

where we have formed the six dimensionless groups

$$
\omega_{X, Y, Z}^{2}=\frac{\tau^{* 2} k_{X, Y, Z}^{*}}{m^{*}}, 2 \lambda_{X, Y, Z}=\frac{\tau^{*} \beta_{X, Y, Z}^{*}}{m^{*}},
$$

and where

$$
\xi_{Y}=\left\{x_{T}^{2}+\left[y_{A}-y_{T}+\left(R^{2}-x_{A}^{2}-z_{A}^{2}\right)^{\frac{1}{2}}\right]^{2}+z_{T}^{2}\right\}^{\frac{1}{2}}-1,
$$

and it is to be understood that the variables appearing explicitly in the governing equations are now dimensionless. In particular, $R$ is the ratio of the dimensional rod length $R^{*}$ to the dimensional natural length $l_{Y}^{*}$ of spring $s_{Y}$. Equations (5-9) are the focus of the rest of this paper. In the next section we show that in the limit of small lateral and transverse tumour movement the equations decouple to leading order.

\subsection{Limit of small lateral and transverse motion}

Here we consider the limit of tumour motion displaying small-amplitude displacements in the $x$ - and $z$-directions, while having order unity displacement in the main $y$-direction. For $\varepsilon \ll 1$ we take the orders of magnitude to be as follows

$$
\begin{gathered}
x_{T} \sim z_{T} \sim \varepsilon, y_{T} \sim 1 ; \quad \theta_{X} \sim \theta_{Z} \sim \varepsilon ; \quad \omega_{X}^{2} \sim \omega_{Z}^{2} \sim \varepsilon, \omega_{Y}^{2} \sim 1 ; \\
\lambda_{X} \sim \lambda_{Y} \sim \lambda_{Z} \sim 1 .
\end{gathered}
$$

Note that in this way springs $s_{X}, s_{Z}$ have low stiffness, enabling them to easily stretch as the tumour moves along a sup-inf line, but lateral movement is damped by the large size of the dashpot resistances in the $x$-, $z$-directions relative to the spring stiffnesses. For order 
unity times, and for order unity values of all three components of the abdominal signal (so that the signal is still fully $3 \mathrm{D}$ ), we expand as follows

$$
\begin{gathered}
x_{T}=\varepsilon x_{1}+\varepsilon^{2} x_{2}+O\left(\varepsilon^{3}\right), \\
y_{T}=y_{0}+\varepsilon y_{1}+O\left(\varepsilon^{2}\right), \\
z_{T}=\varepsilon z_{1}+\varepsilon^{2} z_{2}+O\left(\varepsilon^{3}\right) .
\end{gathered}
$$

Formally, we should also expand the spring angles and model parameters in line with Equation (10). Here, we simply take

$$
\begin{aligned}
& \theta_{X}=\varepsilon \Theta_{X}, \theta_{Z}=\varepsilon \Theta_{Z} \\
& \omega_{X}^{2}=\varepsilon \Omega_{X}^{2}, \omega_{Z}^{2}=\varepsilon \Omega_{Z}^{2},
\end{aligned}
$$

where $\Theta_{X, Z} \sim \Omega_{X, Z} \sim 1$.

The leading order balances of Equation (5) (to $O(\varepsilon)$ ), Equation (6) (to $O(1)$ ), and Equation (7) (to $O(\varepsilon)$ ) are

$$
\begin{aligned}
& \ddot{x}_{1}=\omega_{Y}^{2}\left[\left|y_{A}-y_{0}+\left(R^{2}-x_{A}^{2}-z_{A}^{2}\right)^{\frac{1}{2}}\right|-1\right] \Theta_{X}-2 \lambda_{X} \dot{x}_{1}, \\
& \ddot{y}_{0}=-\omega_{Y}^{2}\left[\left|y_{A}-y_{0}+\left(R^{2}-x_{A}^{2}-z_{A}^{2}\right)^{\frac{1}{2}}\right|-1\right]-2 \lambda_{Y} \dot{y}_{0}, \\
& \ddot{z}_{1}=\omega_{Y}^{2}\left[\left|y_{A}-y_{0}+\left(R^{2}-x_{A}^{2}-z_{A}^{2}\right)^{\frac{1}{2}}\right|-1\right] \Theta_{Z}-2 \lambda_{Z} \dot{z}_{1} .
\end{aligned}
$$

The main point is that Equation (17), governing the order unity motion in the $y$ direction, has effectively decoupled from the other equations, in that Equation (17) can be solved - subject to a known abdominal breathing signal - independently for $y_{0}$. This solution can then be inserted into Equations $(16,18)$, which can be solved separately should lateral and transverse corrections of $O(\varepsilon)$ be required. A higher-order balance of Equation (6) would give $y_{1}$, an $O(\varepsilon)$ correction to the motion in the $y$-direction.

In clinical settings the amplitude of tumour motion in the $y$-direction is typically significantly larger than that in the $x$ - and $z$-directions [17]. For this reason we take the analysis of this section to support our work in the subsequent sections, which concentrate on a 1D-3D model. This refers to solving the one-dimensional Equation (17) subject to a fully 3D abdominal breathing motion. Physically, the solution of this model is the dominant component of the fully 3D tumour motion in the limit of small lateral and transverse displacements.

First, in Section 3, we validate the numerical approach by comparing sample data with analytical predictions obtained by taking limiting cases of the key parameters in Equation (17). In Section 4 we will use a numerical scheme to solve Equation (17) with patientspecific parameters determined by an optimization routine.

\section{Analytical validation of the numerics}

\subsection{The 1D-3D model}

For clarity we reiterate that Equation (17) governs the dominant tumour motion, along the sup-inf line, driven by a full $3 \mathrm{D}$ and time-dependent signal from the abdomen motion 
$\left(x_{A}, y_{A}, z_{A}\right)$. The abdomen motion is used because external tracking of it is relatively easy, as opposed to the difficulty of obtaining real-time measurements of the diaphragm position.

Our task in this section is to show analytically that this equation has physically reasonable solutions under certain circumstances (or limiting parameter ranges), or that under those circumstances predictions can be made which agree with numerical solutions. Since in a phenomenological and non-dimensional model of this kind we have no a priori notion of 'large' and 'small' values of the key parameters, a feel for the size of these parameters will also be obtained here. This work jointly provides confidence in the model and in the numerical approach.

Numerical modelling was carried out in Matlab (Mathworks, Natick, MA, USA). In this testing section of the paper, we generate a realistic but simplified breathing signal; real clinical data is used in Section 4. The $z$-component of the respiratory signal was simulated by the Weibull distribution [21] given by

$$
z_{A}(t)=\frac{\sigma}{\eta}\left(\frac{T-\gamma}{\eta}\right)^{\sigma-1} \mathrm{e}^{-\left(\frac{T-\gamma}{\eta}\right)^{\sigma}}
$$

for $t$ modulo 4, and where $\eta$ is a scale parameter, $\sigma$ is a shape (or slope) parameter, and $\gamma$ is a location parameter. A representative respiratory signal was found for $\sigma=4, \eta=2$ and $\gamma=0$ and will be used throughout Section 3. For convenience, $x_{A}$ and $y_{A}$ were set to zero. Since for this section we set the ratio $R$ to be unity, we must take $z_{A} \leq 1$ otherwise Equation (17) is violated. (This is a minor aspect of the non-dimensional model - future clinical developments of the model will be dimensional.) Therefore, $z_{A}$ was scaled and offset such that the respiratory signal was in the appropriate range. The scaled respiratory signal was applied to Equation (17) as the driving term, and the equation was solved with an in-built Matlab function for non-stiff differential equations [3].

\subsection{Analysis of the 1D-3D model}

The key physical parameters are the dimensionless group $\omega_{Y}^{2}$ representing the spring stiffness, and the dimensionless group $\lambda_{Y}$ representing the damping coefficient. The relative orders of magnitude of these terms, and of the tumour motion $y_{0}$ are examined as follows.

\subsection{1 $\lambda_{Y} \sim \omega_{Y} \sim \ddot{y}_{0}-$ the initial balance}

In this situation no straightforward analysis can be performed and we must in general resort to numerical solutions. Broadly, we can say that when $\omega_{Y}>\lambda_{Y}$ the motion is underdamped, suggesting potential for spurious oscillations imposed on the solution; when $\omega_{Y}<\lambda_{Y}$ it is overdamped, meaning such oscillations should be absent, although over-damping could prevent any motion; and when $\omega_{Y}=\lambda_{Y}$ the motion is critically damped.

Selected results of numerical experiments to solve Equation (17) are shown in Figure 2. In Figures 2(a)-(c) three different values for $\omega_{Y}=\lambda_{Y}$ are shown highlighting the effect of the magnitude of the parameters on the predicted tumour position. The evolution of the tumour signal for values of $\omega_{Y}=\lambda_{Y}$ in the range from 0.1 to 100 is shown in Figure 2(d). Both the amplitude of the predicted tumour position and the lag between it and the abdominal signal increase with the magnitudes of $\omega_{Y}=\lambda_{Y}$. Figure 2(e)-(f) shows the tumour signal for $\omega_{Y}$ larger than $\lambda_{Y}$ and smaller than $\lambda_{Y}$, respectively.

\subsection{2 $\omega_{Y}^{2} \ll 1-$ the weak spring}

Let $\omega_{Y}^{2}=\varepsilon \Omega_{Y}^{2}$ for $\varepsilon \ll 1$ and $\Omega_{Y} \sim 1$. Now, since $t, x_{A}, y_{A}, z_{A}, R$ are always of order unity ${ }^{1}$, and since $y_{0}$ is also of $O(1)$, we must have $\lambda_{Y} \sim 1$ here. Thus Equation (17) has leading 

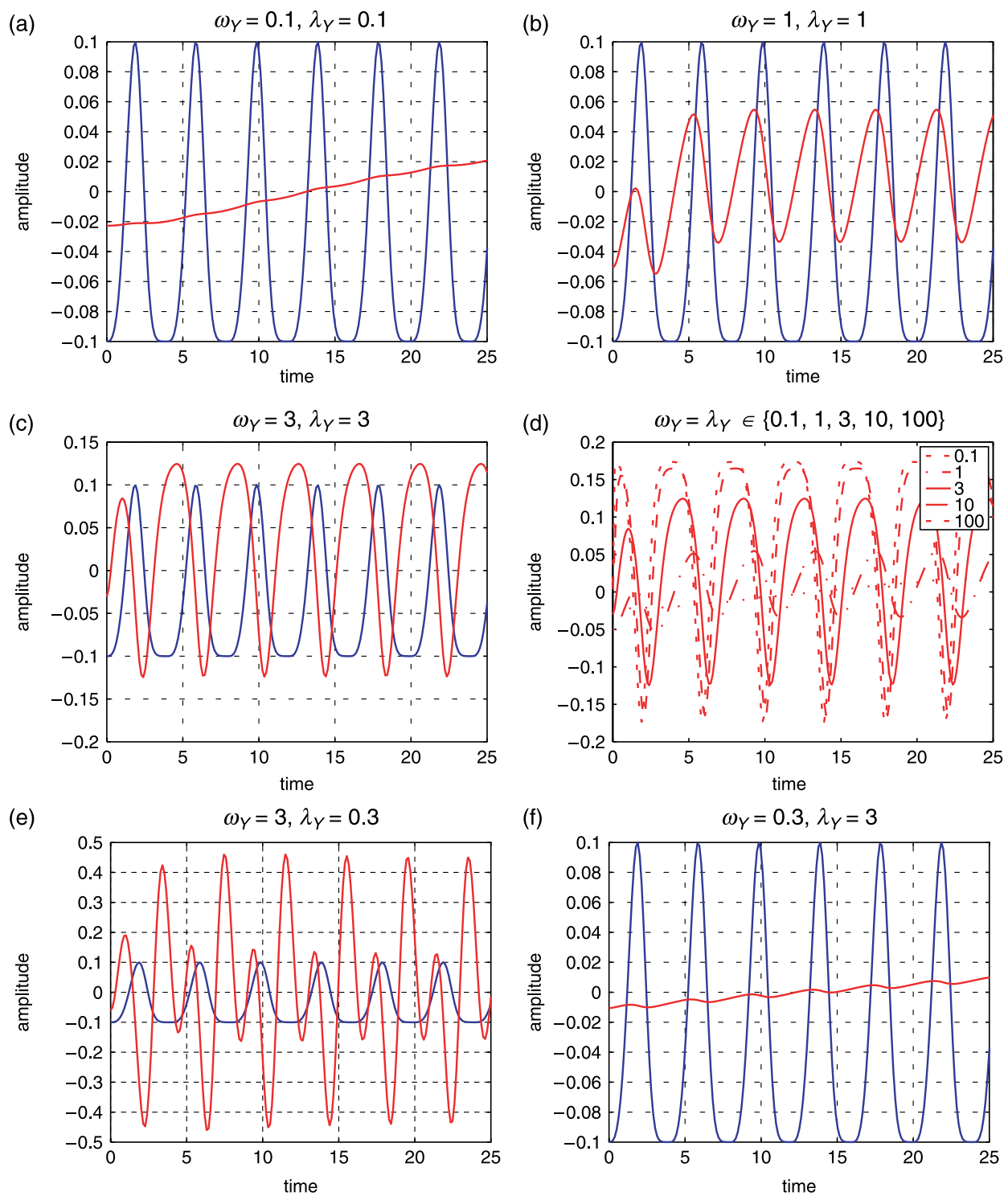

Figure 2. Numerical solutions of Equation (17) for different values of $\omega_{Y=} \lambda_{Y}$ in (Figures 2(a)(d)), $\omega_{Y}>\lambda_{Y}$ in (Figure 2(e)), and for $\omega_{Y<} \lambda_{Y}$ in (Figure 2(f)). The blue line represents the abdominal respiratory signal $z_{A}$ and the red line the leading order sup-inf tumour position $y_{0}$. For comparison the respiratory signal is superimposed on the tumour signal such that $\bar{z}_{A}=0$.

order balance

$$
\ddot{y}_{0}+2 \lambda_{Y} \dot{y}_{0}=0
$$

which has the general solution

$$
y_{0}=A \mathrm{e}^{-2 \lambda_{Y} t}+B
$$

for constants $A, B \in \mathbb{R}$ dependent on the initial conditions. This suggests that $y_{0} \rightarrow B$ for large times, as is seen to occur in the numerical solution of Equation (17) shown in 

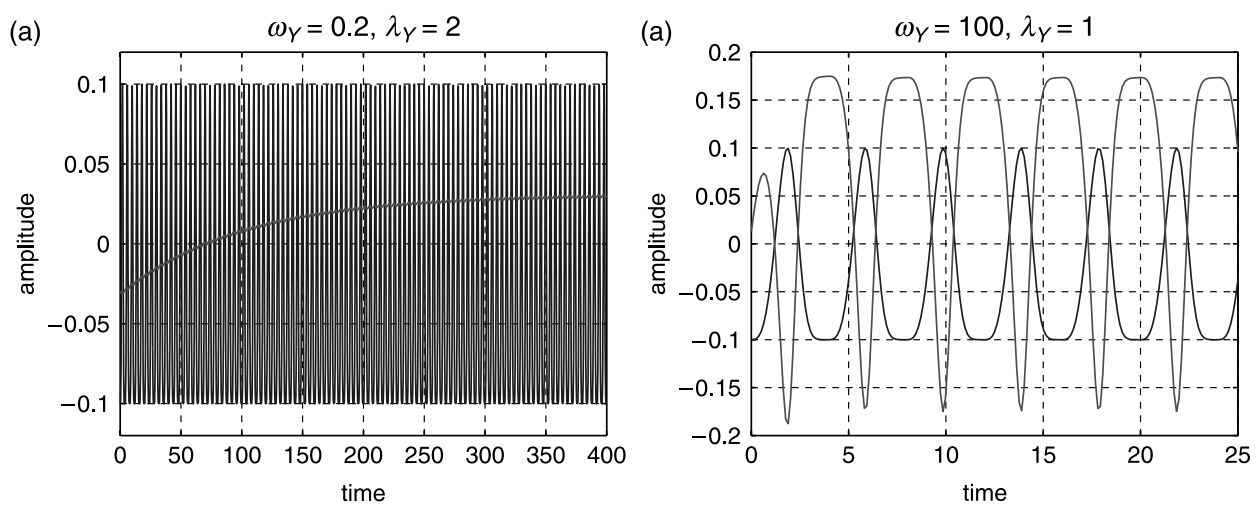

Figure 3. Numerical solutions of Equation (17) for a weak spring (a) and a stiff spring (b).

Figure 3(a). In this particular case for a signal of period 4, 'large' times are those of order 100 periods, and this is only known a posteriori.

Moreover, the limiting case of setting $\omega_{Y} \equiv 0$ gives the numerical solution $y_{0} \equiv B$ for all time, as would be expected: if the spring stiffness is (close to) zero, or the mass of the tumour is (approaching) infinity ${ }^{2}$, the tumour position will not change.

\subsection{3 $\omega_{Y}^{2} \gg 1-$ the stiff spring}

With all other orders of magnitude being the same as in Section 3.2.2, the dominant balance of Equation (17) is

$$
\left|y_{A}-y_{0}+\left(R^{2}-x_{A}^{2}-z_{A}^{2}\right)^{\frac{1}{2}}\right|-1=0,
$$

such that

$$
y_{0}=y_{A}+\left(R^{2}-x_{A}^{2}-z_{A}^{2}\right)^{\frac{1}{2}} \pm 1 .
$$

This suggests a rigid-body motion, with the tumour motion exactly following the diaphragm motion, either near $y=0$ or near $y=-2$, with the latter being unrealistic. Physically this makes sense, as a stiff spring approximates a rigid rod (equivalently in this nondimensional system, a very light tumour has low inertia). The spring is dominant and damping almost irrelevant. This can be seen in the numerical solutions presented in Figure 3 (b), in which the graph of the tumour motion is in anti-phase with the graph of the abdomen position - as the abdomen rises, the tumour is pulled in rigid-body motion towards the descending diaphragm, and conversely.

\subsection{4 $\lambda_{Y} \ll 1-$ light damping}

While $\omega_{Y}^{2} \sim 1$ and all other parameters apart from $\lambda_{Y}$ are the same size as in Section 3.2.2, setting $\lambda_{Y}=\varepsilon \Lambda_{Y}$ for $\varepsilon \ll 1$ and $\Lambda_{Y} \sim 1$ gives the leading order balance of Equation (17) to be

$$
\ddot{y}_{0}+\omega_{Y}^{2}\left[\left|y_{A}-y_{0}+\left(R^{2}-x_{A}^{2}-z_{A}^{2}\right)^{\frac{1}{2}}\right|-1\right]=0
$$

which is the equation of a forced, undamped spring. The solutions of such a system can blow up due to positive feedback from the forcing, suggesting that small values of $\lambda_{Y}$ should not be 
(a)

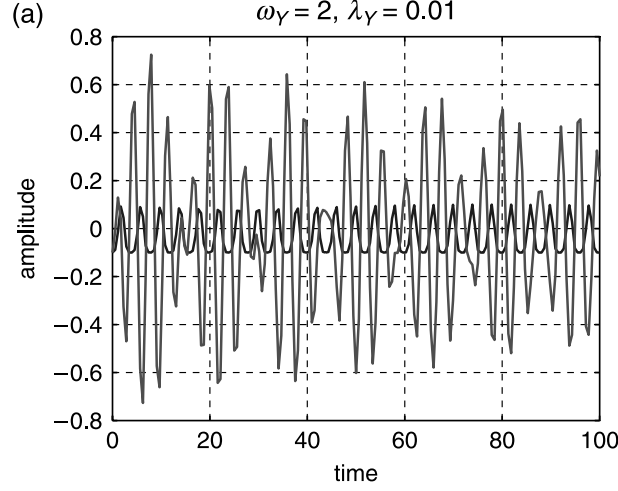

(b)

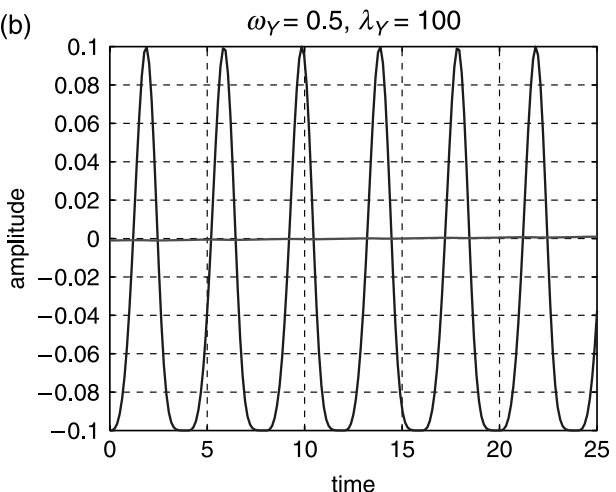

Figure 4. Numerical solutions of Equation (17) for light damping (a) and heavy damping (b).

chosen in the numerical routine (although it may be admissible to allow $O(1)$ values of $\lambda_{Y}$ such that $\lambda_{Y} \ll \omega_{Y}^{2}$ ). Although the solution shown in Figure 4(a) does not blow up, large spurious oscillations occur due to the underdamping.

\subsection{5 $\quad \lambda_{Y} \gg 1-$ heavy damping}

With all other orders of magnitude as in Section 3.2.4, the dominant effect is $\dot{y}_{0}=0$ : no tumour motion (to leading order). This is seen in numerical solutions shown in Figure 4(b), and is to be expected, since very heavy resistance to motion, or a very light tumour with $O(1)$ resistance ${ }^{3}$, effectively prevents the tumour from moving.

Having gained some confidence in both the model and the general numerical approach, we proceed in Section 4 to model real tumour trajectories obtained from clinical data.

\section{Applying the model to clinical patient data}

\subsection{The data-collection process}

The clinical data we used is based on the Wuerzburg Adaptive Tumour Tracking System (ATTS), which is a collaborative project between the University of Wuerzburg, Germany and Medical Intelligence, Schwabmuenchen, Germany, an industrial collaborator [12]. Figure 5 shows a schematic overview of the ATTS. A detailed description of the data acquisition process can be found elsewhere [22]. The purpose of the ATTS is to compensate tumour motion in real time by repositioning the patient relative to the stationary radiation beam. The patient is placed on a robotic couch with six degrees of freedom and submillimetre positioning accuracy [13]. Dynamic repositioning with the hexapod table is initiated and guided by both the respiratory signal and, to some extent, by continuous imaging of the chest with the therapy beam. It is because the imaging information is less reliable (due to poor image quality) that sophisticated models are required to deduce the tumour position from the respiratory signal. The respiratory signal is obtained by tracking optical markers on the patient's abdomen by an in-room infrared tracking camera with submillimetre precision and at a frequency of approximately $20 \mathrm{~Hz}$. Tumour information is acquired simultaneously by automatically tracking the tumour in images from the electronic portal imaging device (EPID), acquired at a rate of approximately $3 \mathrm{~Hz}[11]$. 


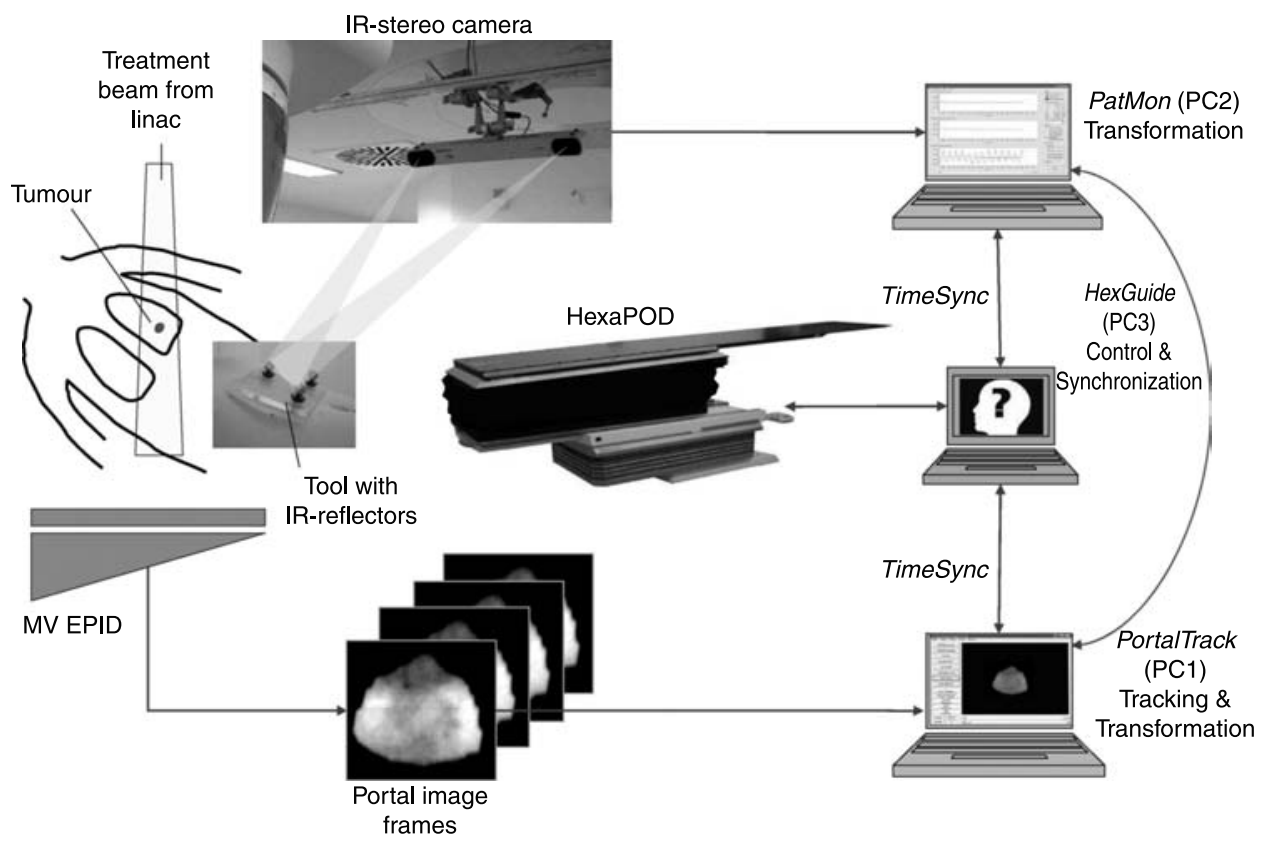

Figure 5. A sketch of the Wuerzburg Adaptive Tumour Tracking System, indicating the data acquisition process. The abdominal respiratory signal is acquired with an infra-red (IR) stereo realtime tracking camera and the tumour signal is obtained by tracking the tumour in portal images [11].

\subsection{Preprocessing of the clinical data}

The conventions of the physical model as shown in Figure 1 are such that the tumour oscillates around the origin. The location of the respiratory signal is some distance away in the direction of the negative $y$-axis. In order to apply the dimensionless equations to the clinical data some scaling, offsetting and resampling of the raw data was required. For example, the term $\left(R^{2}-x_{A}^{2}-z_{A}^{2}\right)$ in Equation (17) must be positive, and so the respiratory signal had to be scaled down. As a consequence the model output, namely the amplitude of the tumour trajectory, was restricted to certain values requiring the tumour trajectory to be scaled as well. The clinical data set selected was that of a patient during stereotactic lung radiotherapy treatment [23]. To demonstrate the principle, we chose a patient who exhibited regular breathing with a tumour which was relatively 'visible' in the portal images. The main direction of abdominal movement was in the $z$-direction, and the main direction of the tumour motion was in the $y$-direction, as shown in Figure 6. The tumour movement displayed only small lateral and transverse motion, a requirement of the 1D-3D model.

\subsection{Results of modelling the clinical data}

As mentioned previously, the model equations are generic for modelling the relationship between abdominal motion and the lung tumour position. Differences in the dynamics of the tumour motion may originate from a number of different reasons, such as the tumour location or the breathing technique of a particular patient. The spring-dashpot system can model this by means of adjusting the dimensionless spring stiffness $\omega_{Y}^{2}$ and the value of the dimensionless damping factor $\lambda_{Y}$ of the dashpot. Also, the ratio of $l_{y}^{*}$ and the rod length $R^{*}$, i.e. $R$, has an impact on the amplitude of the model output. 


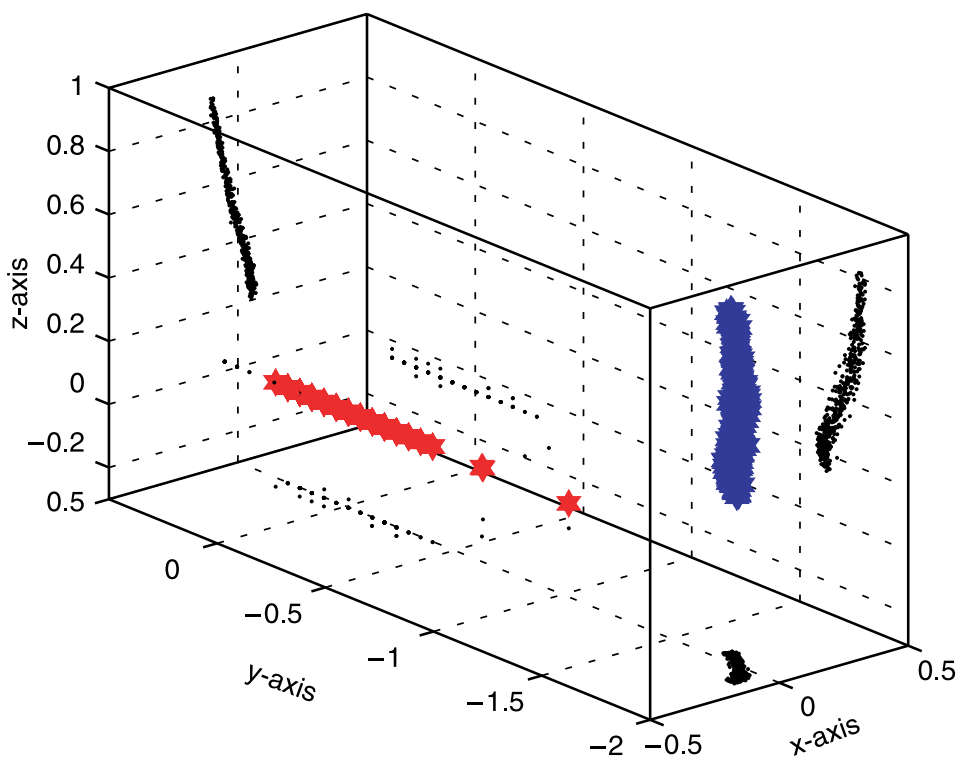

Figure 6. Spatial distribution of clinical respiratory data (blue) and corresponding tumour data (red). The data are also projected onto the $(x, y)-,(x, z)-$, and $(y, z)$-planes (black).

The 3D components of the respiratory signal shown in Figure 6 and used to drive the 1D-3D model are shown as functions of time in Figure 7(a). Initial parameters were optimised manually in order to get reasonable agreement between the measured tumour signal, represented by black dots and a dashed line in Figure 7(b), and the model output. Using Matlab, an in-built unconstrained non-linear search algorithm [8] was applied to fine-tune $\omega_{Y}^{2}$ and $\lambda_{Y}$. The cost function utilized was based on the root-mean-square (rms) error between the measured tumour signal and the model output. The optimization found a minimum for $\omega_{Y}^{2}=6.4$ and $\lambda=7.5$. The resulting tumour model output is shown in 4.3 as red dots and a red line. The rms difference in this non-dimensional scaled system was $6.4 \%$.

(a)

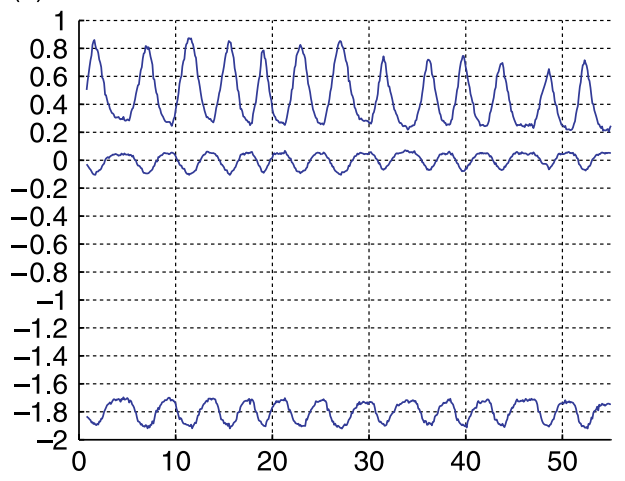

(b)

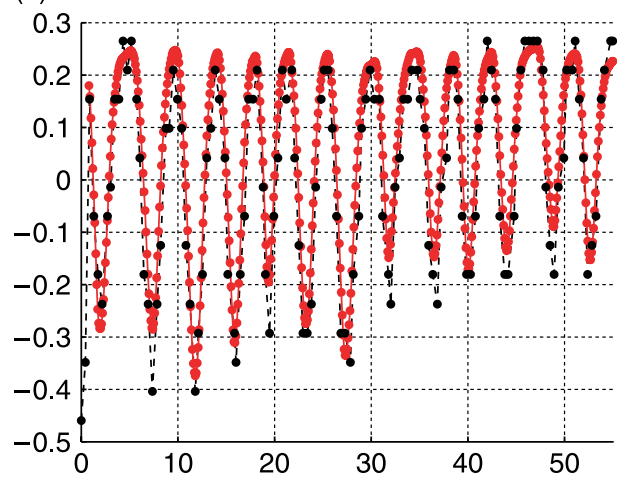

Figure 7. Matlab simulations: clinical data and modelling result. (a) The individual components, $x_{A}$ (middle), $y_{A}$ (bottom), and $z_{A}$ (top) of the respiratory signal as a function of time. (b) Calculated and measured tumour signal $y_{T}$ as a function of time. The black dots correspond to the measured data and the red dots correspond to the calculated data for $y_{0}$. 
The results indicate that the spring-dashpot approach is indeed capable of closely modelling the correlation between clinical abdominal motion and measured tumour position. The 1D-3D model parameters can automatically be optimized using a simple cost function. In clinical terms, there are two important aspects of the model output to consider: the lag and the signal local extrema. A persistent phase shift, or lag, between the model output and the measured tumour signal would lead to a systematic tracking error, inevitably resulting in underdosing of the tumour during radiotherapy treatment. Figure 7(b) shows that there is excellent agreement between the phase of both signals.

By contrast, small disagreements at the local extrema have considerably less impact on tumour dose due to the short residency time of the tumour close to local extrema. In Figure 7(b), minor differences can be seen between some of the local extrema of the two signals which are likely to have minimal impact $[5,16]$. It should also be noted that the sample data from the ATTS are based on automatic tracking of the tumour position in portal images. [1] discussed the technical details of the image acquisition process. In particular, it is obvious that a low sampling rate of tumour position introduces some error, which is likely to be worse when a local extremum falls between two sampling points. The data from the ATTS used in this work were based on a sampling rate of $3 \mathrm{~Hz}$ for the tumour. A higher experimental sampling rate would possibly improve the numerical agreement between measured and modelled tumour positions as indicated by the rms difference.

\section{Conclusions}

A spring-dashpot system was presented which models the physical relationship between abdominal breathing motion and lung tumour motion during radiotherapy. The tumour was modelled as being attached to three springs and three dashpots, one spring-dashpot pair for each room coordinate, and reflecting the mechanical and dynamical properties of human anatomy. One spring was additionally attached to a point representing the diaphragm, which in turn was attached by a rigid rod to a point representing the abdomen. This point is free to move in a fully 3D way. The coupled dimensional equations of motion were nondimensionalised, yielding the 3D-3D model: three coupled equations describing the 3D motion of the system forced by a 3D abdominal breathing signal. The input to this model is a 3D breathing signal; the parameters of the model need to be optimised for each patient; and the output is a modelled lung tumour motion. A numerical scheme was introduced to solve a clinically-relevant limiting case of the 3D-3D model, namely when lateral and transverse tumour motions are small. This 1D-3D model was obtained by asymptotic analysis of the 3D-3D model. It was found that the equation governing the dominant component of the tumour motion decouples from the lateral and transverse equations of motion. Analysis of the 1D-3D model was compared with sample results of a numerical scheme written to solve the model. This numerical data was obtained with a quasi-realistic breathing signal, and was also used to estimate parameter ranges.

Our main aim was to investigate whether such an approach was suitable for modelling the spatial relationship between abdominal motion, the input, and lung tumour motion, the output. Real clinical data, reflecting the conditions of the 1D-3D model, and obtained from a prototype tumour tracking system, provided the input abdominal signal. Model parameters were found by minimising a cost function. The results were in excellent agreement with the measured tumour motion, in particular showing very close phase matching, which is of clinical importance.

We anticipate that such models will be superior to more commonly used 'grey box' approaches, such as previously-studied least-square models, which is currently under 
investigation. Future work will aim towards fully 3D-3D numerical simulations using clinical data and address the practical aspects of implementing such an approach in a clinical environment.

\section{Acknowledgements}

The authors would like to thank Dr Juergen Wilbert and Kurt Baier from the University of Wuerzburg, Germany, for providing clinical data sets, and the referee for helpful comments.

\section{Notes}

1. Note that $t \sim 1$ assumes that there are no short or long time scales; no rapid changes, and no longer-term changes are considered.

2. Recall that the dimensionless group $\omega_{Y}$ is proportional to spring stiffness $k_{Y}^{*}$ and inversely proportional to tumour mass $m^{*}$. The 'weak spring' case is equivalent to a 'heavy tumour' case, and likewise for the remaining cases of this section.

3. Recall that the dimensionless group $\lambda_{Y}$ is proportional to friction coefficient $\beta_{Y}^{*}$ and inversely proportional to the tumour mass $m^{*}$.

\section{References}

[1] K. Baier and J. Meyer, Fast image acquisition and processing on a TV camera-based portal imaging system, Z. Med. Phys. 15 (2005), pp. 1-4.

[2] R.I. Berbeco, et al., Towards fluoroscopic respiratory gating for lung tumours without radiopaque markers, Phys. Med. Biol. 50 (2005), pp. 4481-4490.

[3] J. Dormand and P. Prince, A family of embedded Runge-Kutta formulae, J. Comput. Appl. Math. 6 (1980), pp. 19-26.

[4] W.D. D'Souza, S.A. Naqvi, and C.X. Yu, Real-time intra-fraction-motion tracking using the treatment couch: a feasibility study, Phys. Med. Biol. 50 (2005), pp. 4021-4033.

[5] M. Engelsman, et al., How much margin reduction is possible through gating or breath hold?, Phys. Med. Biol. 50 (2005), pp. 477-490.

[6] ICRU50, Prescribing, recording and reporting photon beam therapy, Tech. Rep. ICRU Report 50, Bethesda, MD, USA (1993).

[7] P.J. Keall, et al., Four-dimensional radiotherapy planning for DMLC-based respiratory motion tracking, Med. Phys. 32 (2005), pp. 942-951.

[8] J.C. Lagarias, et al., Convergence properties of the Nelder-Mead simplex method in low dimensions, SIAM J. Optim. 9 (1998), pp. 112-147.

[9] Ministry of Health, Cancer patient survival 1994-2003, Tech. Rep. ISBN: 0-478-30013-1 (Web), New Zealand Health Information Service, PO Box 5013, Wellington, New Zealand (2006).

[10] J. Meyer, et al., Three-dimensional spatial modelling of the correlation between abdominal motion and lung tumour motion with breathing, Acta Oncol. 45 (2006), pp. 923-934.

[11] J. Meyer, et al., Tracking moving objects with megavoltage portal imaging: a feasibility study, Med. Phys. 33 (2006), pp. 1275-1280.

[12] J. Meyer, et al., On the use of a hexapod table to improve tumour targeting in radiation therapy, in Bioinspiration and Robotics: Walking and Climbing Robot, M. Habib, ed., Advanced Robotic Systems International and I-Tech, Chapter V, Section 30, Vienna: I-Tech Education and Publishing, 529-544.

[13] J. Meyer, et al., Positioning accuracy of cone-beam computed tomography in combination with a hexapod robot treatment table, Int. J. Radiat. Oncol. Biol. Phys. 67 (2007), pp. 1220-1228.

[14] M.J. Murphy, Tracking moving organs in real time, Semin. Radiat. Oncol. 14 (2004), pp. 91-100.

[15] P. Parikh, et al., Dynamic accuracy of an implanted wireless ac electromagnetic sensor for guided radiation therapy; implications for real-time tumor position tracking, Med. Phys. 32 (2005), pp. 2112-2113. 
[16] E. Rietzel, et al., Design of 4D treatment planning target volumes, Int. J. Radiat. Oncol. Biol. Phys. 66 (2006), pp. 287-295.

[17] Y. Seppenwoolde, et al., Precise and real-time measurement of 3D tumor motion in lung due to breathing and heartbeat, measured during radiotherapy, Int. J. Radiat. Oncol. Biol. Phys. 53 (2002), pp. 822-834.

[18] S. Shimizu, et al., Detection of lung tumor movement in real-time tumor-tracking radiotherapy, Int. J. Radiat. Oncol. Biol. Phys. 51 (2001), pp. 304-310.

[19] B. Suki and K.R. Lutchen, Lung tissue viscoelasticity, in Wiley Encyclopedia of Biomedical Engineering, Wiley, New York, 2006, pp. 1-12.

[20] B. Suki, et al., Biomechanics of the lung parenchyma: critical roles of collagen and mechanical forces, J. Appl. Physiol. 98 (2005), pp. 1892-1899.

[21] W. Weibull, A statistical distribution function of wide applicability, J. Appl. Mech. 18 (1951), pp. 293-297.

[22] J. Wilbert, et al., Tumor tracking and motion compensation with an adaptive tumor tracking system (ATTS): system description and prototype testing, Med. Phys. 35 (2008), pp. $3911-3921$.

[23] J. Wulf, et al., Dose-response in stereotactic irradiation of lung tumors, Radiother. Oncol. 77 (2005), pp. 83-87.

[24] J. Zhang, et al., Real time tracking of respiratory tumor motion based on external respiratory output, Med. Phys. 33 (2006), p. 2046. 


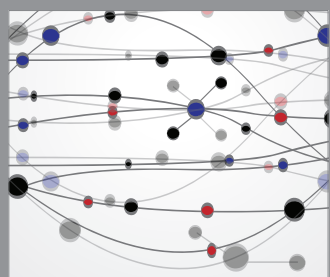

The Scientific World Journal
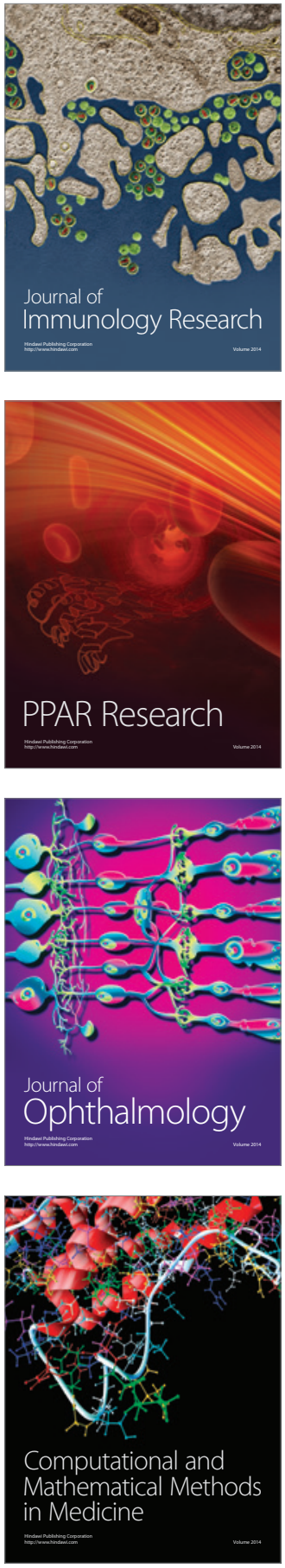

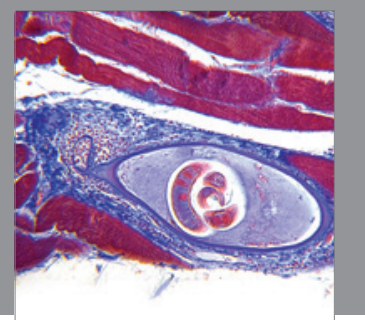

Gastroenterology

Research and Practice
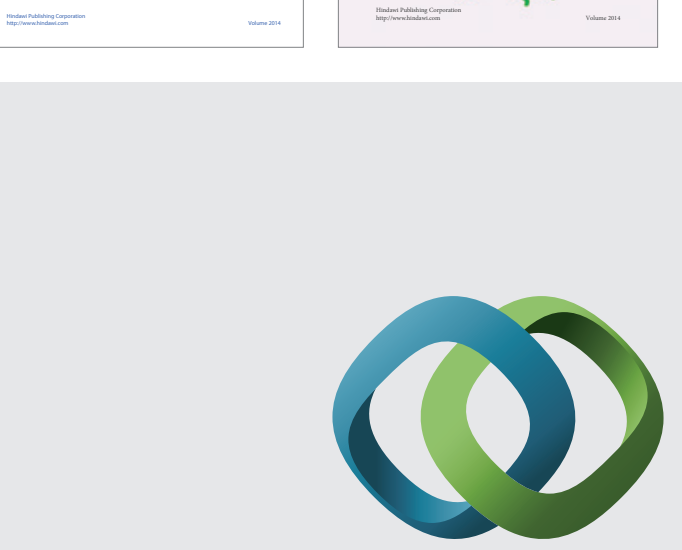

\section{Hindawi}

Submit your manuscripts at

http://www.hindawi.com
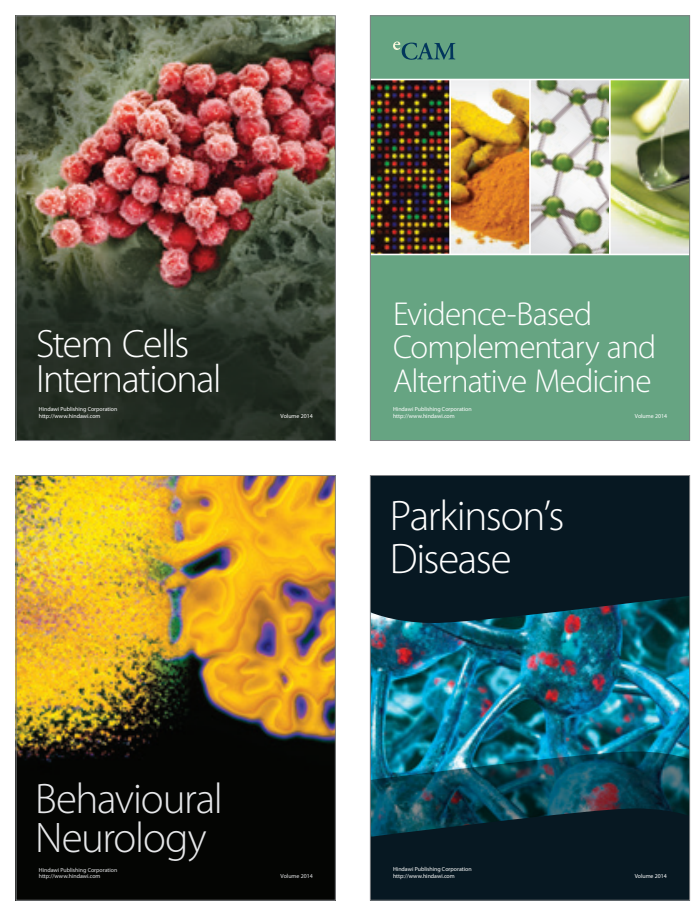

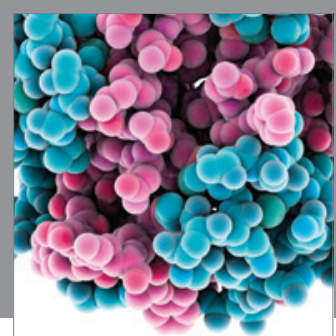

Journal of
Diabetes Research

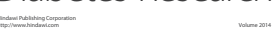

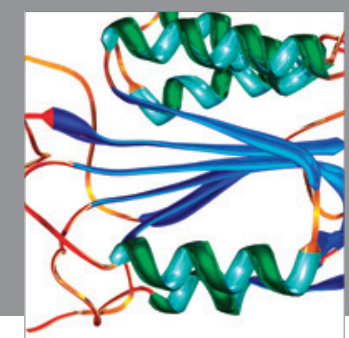

Disease Markers
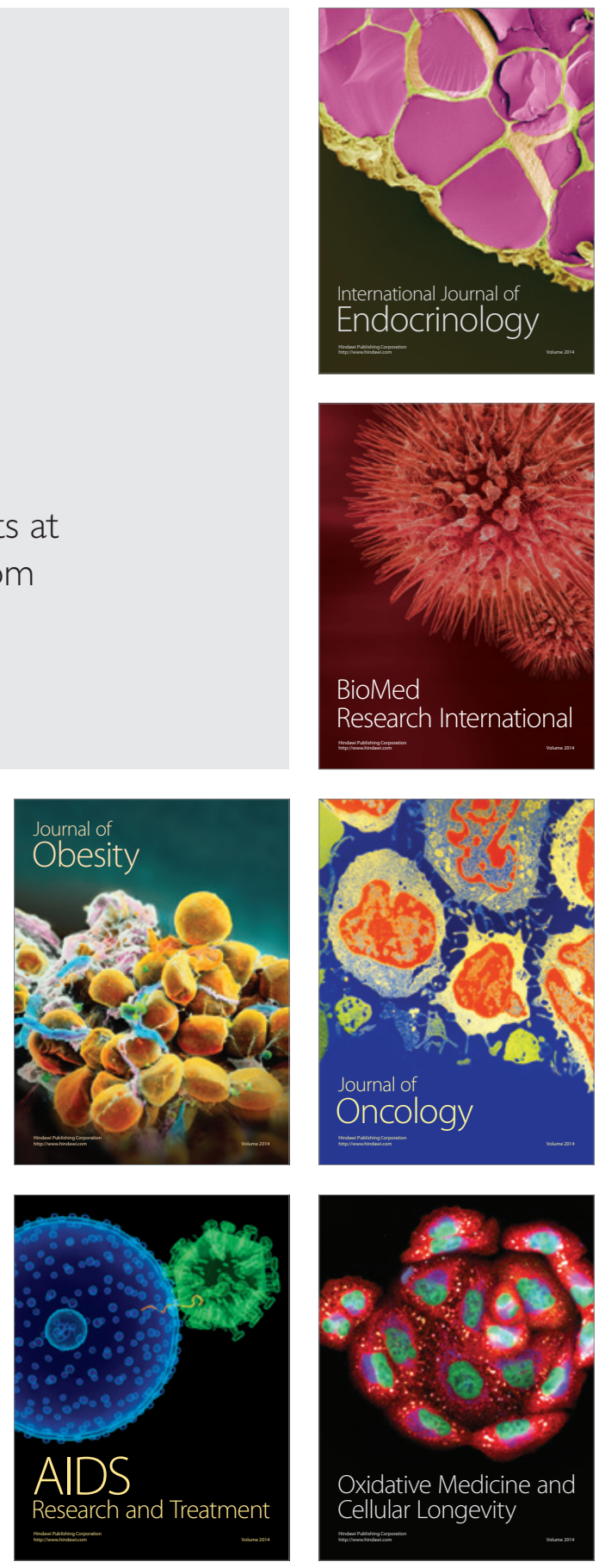